\title{
Cervical total disc replacement with the Mobi-C cervical artificial disc compared with anterior discectomy and fusion for treatment of 2-level symptomatic degenerative disc disease: a prospective, randomized, controlled multicenter clinical trial
}

\author{
Clinical article
}

\author{
Reginald J. Davis, M.D., ${ }^{1}$ Kee D. Kim, M.D., ${ }^{2}$ Michael S. Hisey, M.D., ${ }^{3}$ \\ Gregory A. Hoffman, M.D. ${ }^{4}$ Hyun W. Bae, M.D. ${ }^{5}$ Steven E. Gaede, M.D.,${ }^{6}$ \\ Ralph F. Rashbaum, M.D., ${ }^{3}$ Pierce Dalton Nunley, M.D.,${ }^{7}$ Daniel L. Peterson, M.D. ${ }^{8}$ \\ AND JoHn K. STOKES, M.D. ${ }^{9}$ \\ ${ }^{1}$ Greater Baltimore Neurosurgical Associates, Baltimore, Maryland; ${ }^{2}$ Department of Neurological Surgery, University \\ of California, Davis, Sacramento, California; ${ }^{3}$ Texas Back Institute, Plano, Texas, ${ }^{4}$ Orthopaedics Northeast, Fort \\ Wayne, Indiana; ${ }^{5}$ Cedars Sinai Spine Center, Los Angeles, California; ${ }^{6}$ Oklahoma Brain and Spine Institute, Tulsa, \\ Oklahoma; ${ }^{7}$ Spine Institute of Louisiana, Shreveport, Louisiana; ${ }^{8}$ Austin Brain and Spine, Austin; and ${ }^{9}$ Seton Spine \\ and Scoliosis Center, Austin, Texas
}

\begin{abstract}
Object. Cervical total disc replacement (TDR) is intended to treat neurological symptoms and neck pain associated with degeneration of intervertebral discs in the cervical spine. Anterior cervical discectomy and fusion (ACDF) has been the standard treatment for these indications since the procedure was first developed in the 1950s. While TDR has been shown to be a safe and effective alternative to ACDF for treatment of patients with degenerative disc disease (DDD) at a single level of the cervical spine, few studies have focused on the safety and efficacy of TDR for treatment of 2 levels of the cervical spine. The primary objective of this study was to rigorously compare the Mobi-C cervical artificial disc to ACDF for treatment of cervical DDD at 2 contiguous levels of the cervical spine.

Methods. This study was a prospective, randomized, US FDA investigational device exemption pivotal trial of the Mobi$\mathrm{C}$ cervical artificial disc conducted at 24 centers in the US. The primary clinical outcome was a composite measure of study success at 24 months. The comparative control treatment was ACDF using allograft bone and an anterior plate. A total of 330 patients were enrolled, randomized, and received study surgery. All patients were diagnosed with intractable symptomatic cervical DDD at 2 contiguous levels of the cervical spine between C-3 and C-7. Patients were randomized in a 2:1 ratio (TDR patients to ACDF patients).

Results. A total of 225 patients received the Mobi-C TDR device and 105 patients received ACDF. At 24 months only $3.0 \%$ of patients were lost to follow-up. On average, patients in both groups showed significant improvements in Neck Disability Index (NDI) score, visual analog scale (VAS) neck pain score, and VAS arm pain score from preoperative baseline to each time point. However, the TDR patients experienced significantly greater improvement than ACDF patients in NDI score at all time points and significantly greater improvement in VAS neck pain score at 6 weeks, and at 3, 6, and 12 months postoperatively. On average, patients in the TDR group also maintained preoperative segmental range of motion at both treated segments immediately postoperatively and throughout the study period of 24 months. The reoperation rate was significantly higher in the ACDF group at $11.4 \%$ compared with $3.1 \%$ for the TDR group. Furthermore, at 24 months TDR demonstrated statistical superiority over ACDF based on overall study success rates.

Conclusions. The results of this study represent the first available Level I clinical evidence in support of cervical arthroplasty at 2 contiguous levels of the cervical spine using the Mobi-C cervical artificial disc. These results continue to support the use of cervical arthroplasty in general, but specifically demonstrate the advantages of 2-level arthroplasty over 2-level ACDF. Clinical trial registration no.: NCT00389597 (ClinicalTrials .gov).

(http://thejns.org/doi/abs/10.3171/2013.6.SPINE12527)
\end{abstract}

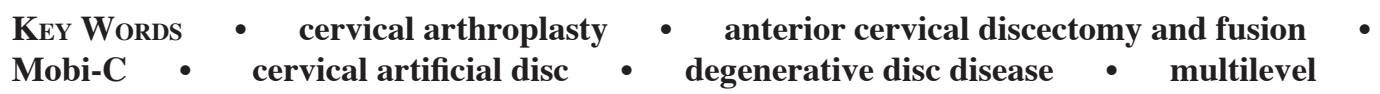

Abbreviations used in this paper: $\mathrm{ACDF}=$ anterior cervical discectomy and fusion; CEC = Clinical End Points Committee; DDD $=$ degenerative disc disease; $\mathrm{HO}=$ heterotopic ossification; $\mathrm{IDE}=$ investigational device exemption; MCS = mental component summary; NDI = Neck Disability Index; NSAID = nonsteroidal antiinflammatory drug; $\mathrm{PCS}=$ physical component summary; ROM = range of motion; SF-12 = 12-Item Short Form Health Survey; TDR $=$ total disc replacement; VAS $=$ visual analog scale.

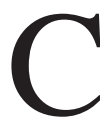
ERVICAL total disc replacement (TDR) is intended to treat neurological symptoms and neck pain associated with degeneration of intervertebral discs in the cervical spine. ${ }^{1,24,41}$ Anterior cervical discectomy and fusion has been the standard treatment for these indications since the procedure was first developed in the 1950s. ${ }^{2,3,18,46}$ Cervical TDR devices were originally developed and implanted in the 1990s and have since become 
part of the continuum of care for cervical degenerative disc disease (DDD) ${ }^{9,49}$ Cervical TDR is an attractive alternative to anterior cervical discectomy and fusion (ACDF) because it achieves neural decompression via an identical anterior surgical approach while preserving mobility and achieving comparable clinical success..$^{25,41,42}$

A primary rationale for the use of TDR has been the hypothesis that by preserving mobility at the operated segment, TDR will allow for normal cervical spine biomechanics and thereby maintain the integrity of adjacent segments. ${ }^{24,41}$ While the goal of ACDF is to eliminate mobility in the treated segment, ${ }^{2,3}$ this loss of mobility has been shown to induce hypermobility and to increase stress and intradiscal pressures on the segments adjacent to fused segments. These factors have been shown to result in greater adjacent-segment degeneration and recurrent radicular symptoms in as many as $25 \%$ of patients. ${ }^{14,23,30,44}$ By maintaining mobility, cervical TDR devices are believed to preserve biomechanics at the adjacent segments, which may slow degeneration and the associated pain and neurological symptoms. ${ }^{6}$ The current literature varies regarding the effect of index-level mobility and the development of adjacent-segment disease, although published studies of the efficacy of cervical TDR have shown a reduction in rates of adjacent-segment degeneration when compared with ACDF.,12,44 In contrast, other studies have shown that the development and/or progression of adjacent-segment degeneration may not be directly linked to the index-level implant, suggesting that perhaps other factors play a significant role in the development of adjacentsegment disease..$^{21,26,31}$

Cervical TDR is available worldwide and has become increasingly common in the US as several TDR devices have moved beyond the investigational stage and been approved for use. ${ }^{22,36,37}$ Several large-scale FDA-regulated investigational device exemption (IDE) clinical trials are in process or have been completed since 2007. These trials have consistently shown that cervical TDR is at least as safe and effective as ACDF for treatment of symptoms associated with cervical DDD. ${ }^{11,13,22,36,37}$ However, these trials compare TDR to ACDF for treatment at a single level only. Patients often suffer from multilevel pathology, and there is evidence that suggests multilevel ACDF is biomechanically more demanding than single-level ACDF. A study by Lopez-Espina et al. ${ }^{32}$ demonstrated that as the number of fused levels increases from 1 to 2, stress on the inferior adjacent intervertebral discs increases during flexion, lateral bending, and torsion by as much as $17 \% .^{28,32}$ In contrast to ACDF, each treated segment in a multilevel TDR is considered biomechanically independent of the adjacent segment. ${ }^{40}$

The biomechanical and surgical differences between patients with single-level pathology and those with multilevel pathology dictate that multilevel TDR be directly compared with multilevel ACDF to evaluate the safety and efficacy of multilevel TDR. The purpose of this study was to use data from the IDE clinical trial of the MobiC cervical artificial disc (LDR Medical) to compare the safety and efficacy of Mobi-C to ACDF for treatment of symptomatic cervical DDD at 2 contiguous levels of the cervical spine.

\begin{abstract}
Methods
Investigational TDR Device

The Mobi-C cervical artificial disc is a low-profile, mobile-bearing TDR device composed of 2 cobalt-chromium alloy endplates and an ultra-high-molecular-weight polyethylene mobile insert. There are no metal-on-metal articulating features; all bearing surfaces are metal on polyethylene. The bone-contacting surfaces of the endplates are plasma-sprayed titanium and hydroxyapatitecoated and feature low-profile, inclined teeth along the lateral edges designed for a bone-sparing surgical technique and to facilitate endplate fixation (Fig. 1). The superior endplate incorporates a convex shape to match the natural cervical anatomy. This mobile-bearing device allows 5 independent degrees of freedom: 2 translational and 3 rotational (Fig. 1). A range of footprints and several heights are available with Mobi-C to satisfy individual anatomical requirements. The device is designed for multilevel applications by eliminating features such as keels, screws, or hardware protruding anterior to the disc space, which accommodates a multilevel surgical technique without risk of interference from adjacent-level implants.
\end{abstract}

\section{Control Device}

The control group received a corticocancellous allograft and an anterior cervical plate utilizing the standard ACDF technique. The allograft was placed in the disc space, and the anterior plate was placed over the graft and secured with screws to the vertebrae according to the manufacturer's published surgical technique. Anterior cervical plate usage was limited to the Slim-Loc Anterior Cervical Plate System (DePuy Spine) and the Sofamor Danek Atlantis or Atlantis Vision Anterior Cervical Plate Systems (Medtronic).

\section{Study Design}

Three hundred thirty patients were randomized, underwent surgery between April 2006 and March 2008 at 24 clinical sites, and comprise the primary analysis population. Patient enrollment required a diagnosis of DDD with radiculopathy or myeloradiculopathy at 2 contiguous levels from C-3 to C-7 that was unresponsive to nonoperative treatment for at least 6 weeks or demonstrated progressive symptoms necessitating immediate surgery. Additional selected inclusion and exclusion criteria are shown in Table 1 . The 330 patients were randomized into either the investigational device group or the control group in a 2:1 ratio, resulting in 225 patients in the investigational (TDR) group and 105 patients in the control (ACDF) group. After confirming a patient's eligibility and having the patient sign an informed consent form, the site investigator or study coordinator called the interactive voice randomization system. Each patient was then assigned to 1 of the treatment groups according to a randomization schedule, which was stratified by preoperative Neck Disability Index (NDI) score and included institutional balancing. This study was registered with the ClinicalTrials. gov database (http://clinicaltrials.gov), and its registration no. is NCT00389597.

This 2-level trial was run concurrently with a separate 


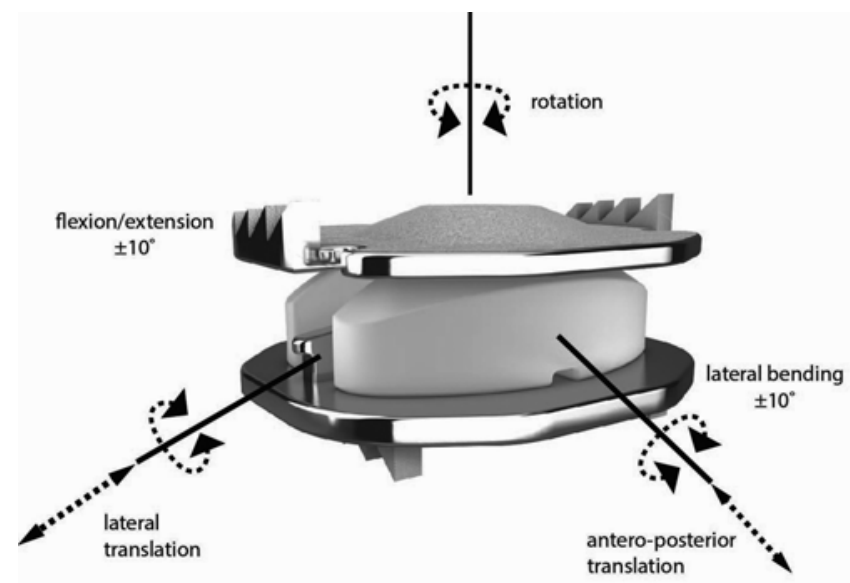

FIG. 1. Mobi-C cervical artificial disc with 2 cobalt-chromium alloy endplates and an ultra-high-molecular-weight polyethylene mobile insert allowing 5 independent degrees of freedom.

study arm to examine safety and efficacy at single-level use. The first qualified patient to enroll at each site, as part of either the single-level or multilevel arm of this clinical trial, was not randomized and was treated with the investigational device to train the principal investigator at the site. This resulted in 9 nonrandomized training patients treated with the TDR device at 2 levels. These patients are not included in the primary analysis population although they were followed and complete patient reports were submitted to the FDA. No statistical differences were found in terms of overall study success between randomized and nonrandomized TDR patients. Masking the physician to the treatment was not possible because the type of implant was evident during surgery. In recent conference proceedings, it was concluded that knowledge of the procedure did not bias the reviewers' radiographic assessments. ${ }^{4}$ Patients remained masked to treatment group assignment until after surgery had been performed to minimize the potential for disproportionate dropouts in either group. There was no concealment of treatment after surgery, because the patient would know the treatment once postoperative radiographs were obtained. As often as possible, objective end points were used to reduce the potential for bias due to postoperative unmasking.

\section{Surgical Technique}

The surgical procedures were performed through a Smith-Robinson anterior approach to the cervical spine. Patients in both groups first underwent a thorough discectomy at both index levels. The disc and any osteophytes were removed. Resection of the posterior longitudinal ligament was left to the operative surgeon's discretion for both treatment groups. Affected neural structures were

\section{TABLE 1: Selected inclusion and exclusion criteria*}

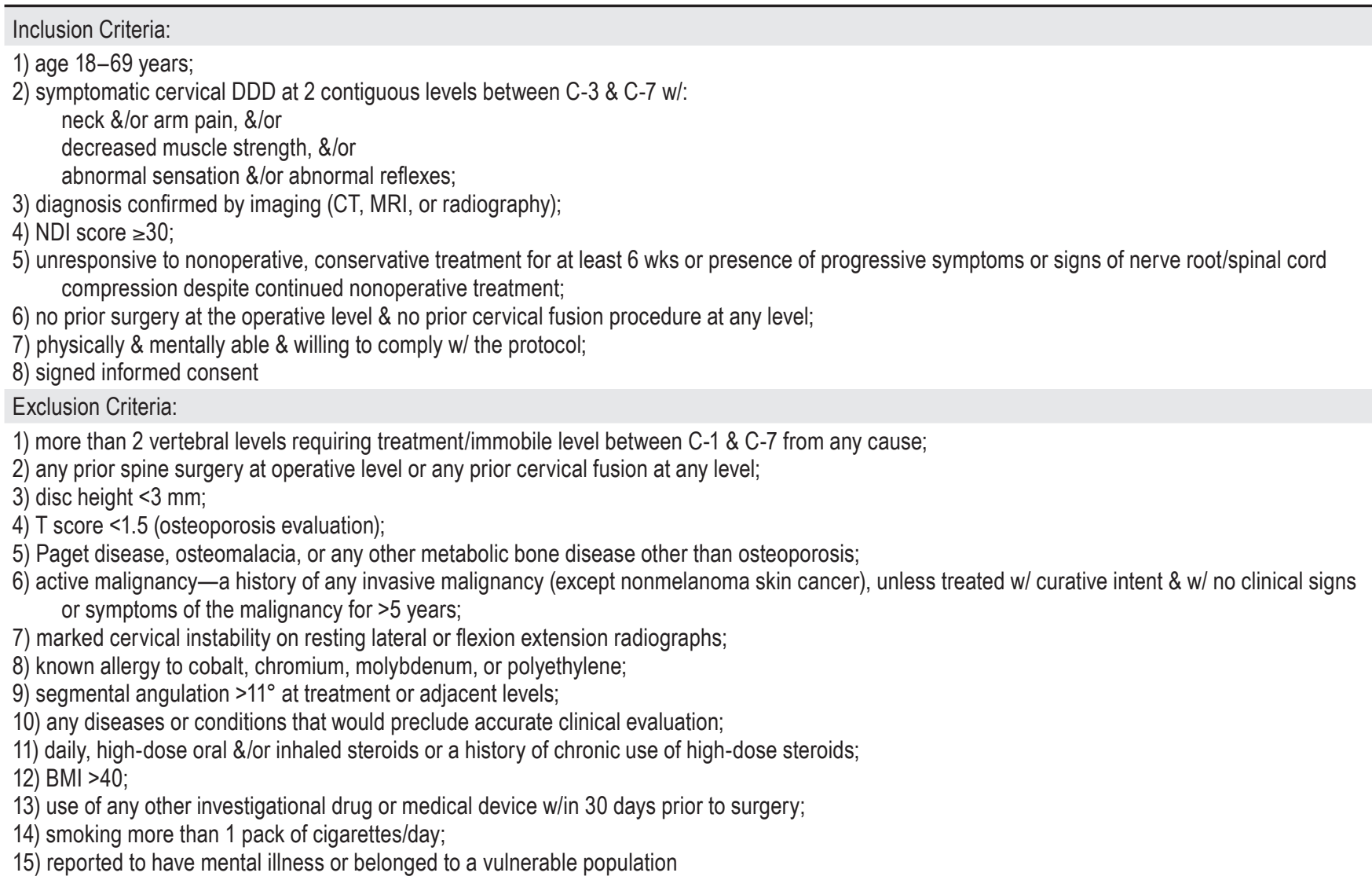

* $\mathrm{BMI}=$ body mass index. 
then decompressed. Either the control device (ACDF) or the investigational device (TDR) was then implanted.

In the TDR group, following discectomy the disc space was distracted in parallel using a vertebral distractor. With disc height restored, distraction was maintained with a Caspar distractor. The cartilaginous endplates were removed to expose the underlying bone with minimal endplate shaping. Using fluoroscopy and the trial instruments provided, the appropriate implant footprint and height were selected. The prosthesis was then inserted into the prepared disc space using a self-retaining inserter. An adjustable stop allowed precise modification of the implant's anteroposterior position. Once final position was obtained, the distraction was released, and slight compression was applied via the Caspar distractor to facilitate seating of the implant teeth into the vertebrae. Anteroposterior and lateral fluoroscopy was used to confirm final implant position (Fig. 2).

\section{Postoperative Care}

While options for rehabilitation were provided in the study protocol, most aspects of postoperative care were left to the treating physician's discretion. All surgeons prescribed and managed a postoperative rehabilitation program for each patient that was aimed at returning the patient to normal activity as soon as possible. As expected, the postoperative care protocol that was prescribed varied somewhat from patient to patient. Consistent with anterior cervical surgery procedures, postoperative care included use of a cervical collar, physical therapy, pain medications, and/or alternative methods. Return to activities of daily living was related to each patient's age, weight, preoperative capabilities, and other factors. Antibiotic coverage was in accordance with the surgeon's standard protocol. Nonsteroidal antiinflammatory drugs (NSAIDs) have been shown to delay bone formation, and evidence suggests that prophylactic use of NSAIDs can reduce the incidence of heterotopic ossification (HO) after other total joint replacement procedures. ${ }^{15,29,34,45}$ To ensure consistency between the study cohorts, all study patients were to stop using NSAIDs 1 week before surgery, and physicians were not to prescribe them prophylactically for at least 3 months after surgery. An exception could be made when $\mathrm{HO}$ was diagnosed in patients in the TDR group. This was a different approach to the prescription of NSAIDs than has been used in other single-level cervical arthroplasty trials, but it allowed this study to achieve a greater level of consistency between the treatment groups, despite potentially putting the TDR group at a disadvantage in the reduction of HO formation. $^{48}$

\section{Clinical Outcome Assessments}

Patients were evaluated preoperatively, 6 weeks postoperatively, and then $3,6,12,18$, and 24 months postoperatively. The primary outcome measure was the overall study success rate of the TDR and ACDF groups at 24 months. These overall success rates-using a composite measure with multiple conditions for success - were used to determine noninferiority and superiority. This clinical trial was prospectively planned and designed by the sponsor (LDR Medical) in conjunction with the FDA. The
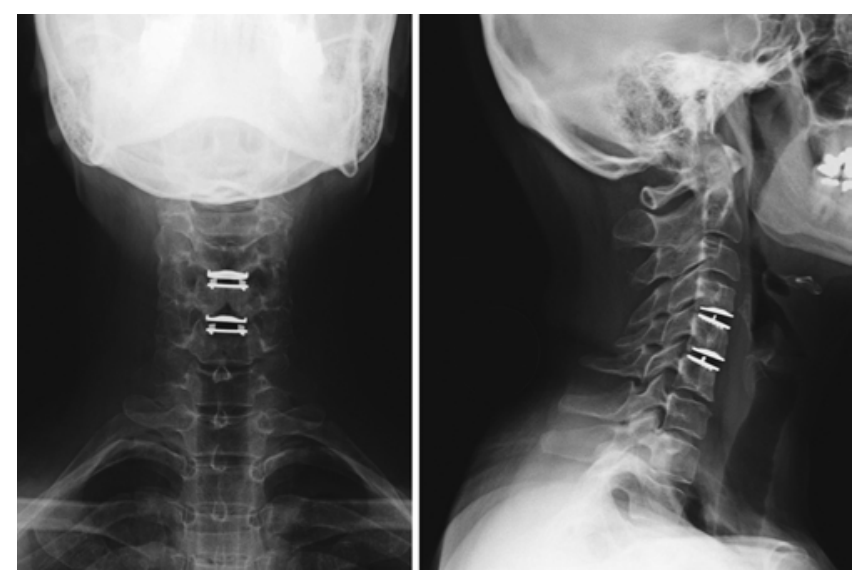

FIG. 2. Anteroposterior (left) and lateral (right) radiographs showing the TDR devices.

protocol was originally submitted to the FDA in October 2005 and received FDA approval in March 2006. At the time of the initiation of this study the selection of a composite measure of overall study success similar to those used in other cervical arthroplasty device trials ${ }^{22,36,37}$ was chosen. The common elements in the definitions of overall study success used in this and other cervical arthroplasty device trials were NDI score, subsequent surgical intervention at the index level or levels, complications, neurological function, and radiographic success. While other measures are certainly of clinical importance (such as the SF-12, patient satisfaction, VAS pain score, ROM, and adjacent segment degeneration), the composite success measure of this trial does not include these as primary outcomes to maintain consistency with other device trials. These outcomes were all collected and analyzed as secondary clinical outcomes.

Overall study success was defined as requiring all 5 of the following metrics: 1) NDI success; 2) no subsequent surgical intervention at the index level or levels; 3) no adverse events assessed by the Clinical End Points Committee (CEC) as major complications; 4) maintenance or improvement in neurological function; and 5) radiographic success.

Neck Disability Index success was defined as an improvement of at least 30 points for patients with a baseline NDI score of at least 60 or as an improvement of at least $50 \%$ from baseline for patients with a baseline NDI score less than 60 . These criteria for NDI success were based on the more rigorous "substantial clinical benefit" criteria as opposed to the more commonly used "minimally clinically important difference" criteria. ${ }^{5}$

Subsequent surgical intervention was defined as any surgery falling into the categories of removal, revision, supplemental fixation, or reoperation. These 4 categories encompassed any surgery that involved modifying the implanted device or devices, or any surgery that was for the original indications at either operative segment. Surgeries at adjacent levels that did not involve the index level or levels were not considered failures, although they were recorded and analyzed.

For the purposes of the study, adverse events were defined as any clinically adverse sign, symptom, syndrome, 
or illness that occurred or worsened during the operative and postoperative period, regardless of causality. Adverse events were assessed by the CEC, which was composed of 3 independent spine surgeons ( 2 orthopedic and 1 neurosurgeon) who were not investigators on the study and did not have any conflict of interest with the sponsor. An adverse event that was determined by the CEC to represent a major complication of the treatment was considered a failure. The adjudication process was performed using a prespecified protocol that included independent reviews by all 3 members, with treatment blinding.

Maintenance or improvement in neurological function was determined based on investigator-conducted tests of reflex, motor, and sensory function.

Radiographic success was included as a component for overall study success to ensure that the implanted device was functioning as intended. The criterion for radiographic success in the TDR group was defined as nonfusion of both treated levels, where nonfusion was defined as at least $2^{\circ}$ of angular motion in flexion/extension or no evidence of bridging trabecular bone across the disc space. Grade IV HO, determined by radiographic assessment, was an indication of bridging bone. Radiographic success in the ACDF group was defined as fusion of both treated levels, less than $2^{\circ}$ of angular motion in flexion/ extension, and evidence of bridging bone across the disc space and radiolucent lines at no more than $50 \%$ of the graft vertebral interfaces.

\section{Radiographic Assessment}

All radiographic analyses were conducted by Medical Metrics, Inc., according to a prespecified radiograph protocol. Neutral lateral, neutral anteroposterior, flexion, extension, left lateral bending, and right lateral bending radiographic views were obtained preoperatively and at all follow-up visits. Quantitative assessments (including angular motion, disc angle, translational motion, disc height, and center of rotation) were made by Medical Metrics using validated proprietary software. ${ }^{43}$ Qualitative assessments of adjacent-segment degeneration, bone bridging, and radiolucency were made by a qualified independent radiologist at Medical Metrics. It was not possible to blind the radiologist to the treatment device. The degeneration of each adjacent segment was determined by the KellgrenLawrence scale of disc degeneration: none (0), minimal (1), definite (2), moderate (3), or severe (4). ${ }^{8,27}$ Assessments were made at baseline and at 12 and 24 months postoperatively. At postoperative time points, a score increase of at least 1 relative to the baseline score indicated adjacentlevel degeneration at that segment. Additional assessments of $\mathrm{HO}$ were made by 2 independent radiologists with a third radiologist to adjudicate in instances of disagreement, using a classification system adapted from McAfee et al. ${ }^{33}$ and Mehren et al. ${ }^{35}$ Each treated segment in the TDR group was graded as follows: $0=$ no evidence of osteophyte formation or $\mathrm{HO} ; \mathrm{I}=\mathrm{HO}$ is detectable from front or side but not in disc space; $\mathrm{II}=\mathrm{HO}$ is growing into disc space, with bone present between planes formed by adjacent endplates, but is not blocking movement; $\mathrm{III}=\mathrm{HO}$ is restricting range of motion (ROM) but some movement or prosthesis remains; or IV = $\mathrm{HO}$ is causing ankylosis and an apparent connection of bridging bone exists between adjacent endplates with no motion between them.

\section{Statistical Analysis}

The primary study hypothesis was noninferiority of the overall clinical success of the investigational group compared with the control group. Noninferiority was tested using an exact $95 \%$ 1-sided confidence bound for the study. If a $10 \%$ noninferiority margin could be ruled out, then superiority was to be tested using an exact $97.5 \%$ 1-sided confidence bound. The closed testing procedure was preplanned to allow for superiority to be tested in the event that noninferiority was established. The overall size of the study and number of patients enrolled were determined based on pilot data and adjusted for $10 \%$ loss to follow-up.

\section{Results \\ Patient Accountability and Demographics}

A total of 347 patients were randomized in the study, 232 to the TDR group and 115 to the ACDF group. Within the randomized population, 225 TDR patients and 105 ACDF patients received study surgery; 17 patients withdrew prior to surgery (Table 2). There were no patients who crossed over from one group to another during study surgery. Additionally, 9 patients were not randomized and were treated with the investigational device as training patients (see Methods). The primary analysis population included the 225 patients in the TDR group and the 105 in the ACDF group who were randomized and received study surgery. The follow-up rates were $98.2 \%$ and $94.3 \%$, respectively, at 24 months. These follow-up rates include all patients with the necessary components for determining overall study success at 24 months. Table 2 shows demographic characteristics of each group and demonstrates that the groups were well balanced with no statistically significant differences.

\section{Surgical Data}

Perioperative data are shown in Table 3 and are presented for all randomized patients and nonrandomized training patients. The 2 treatment groups had statistically similar mean duration of hospitalization $(p=0.23)$ and amount of perioperative blood loss $(p=0.78)$. The operative duration was significantly longer in the TDR group, as expected with any investigational treatment. The mean operative duration in the TDR group was 2.2 hours compared with a mean of 1.8 hours in the ACDF group ( $\mathrm{p}=$ 0.0002).

\section{Visual Analog Scale for Pain}

Patients in both treatment groups had similar VAS pain scores for both neck pain and arm pain at baseline, and patients in both groups showed marked improvement from baseline at all time points (Fig. 3). The mean change in VAS score from baseline was greater in the TDR group than the ACDF group at each time point for both neck pain and arm pain. This difference between groups was statistically significant at 3 months and 6 months postoperatively for neck pain assessments $(\mathrm{p}<0.05)$. No statisti- 
Cervical total disc replacement with Mobi-C at 2 levels

TABLE 2: Patient demographics

\begin{tabular}{|c|c|c|c|}
\hline Demographic Measure & Randomized TDR ( $\mathrm{n}=225)$ & Randomized ACDF $(n=105)$ & $p$ Value ${ }^{*}$ \\
\hline \multicolumn{4}{|l|}{$\operatorname{sex}(\%)$} \\
\hline males & $113(50.2)$ & $45(42.9)$ & 0.2375 \\
\hline females & $112(49.8)$ & $60(57.1)$ & \\
\hline mean age $\pm \mathrm{SD}(\mathrm{yrs})$ & $45.3 \pm 8.10$ & $46.2 \pm 7.99$ & 0.3725 \\
\hline \multicolumn{4}{|l|}{ ethnicity (\%) } \\
\hline Hispanic or Latino & $14(6.2)$ & $7(6.7)$ & $>0.9999$ \\
\hline not Hispanic or Latino & $211(93.8)$ & $98(93.3)$ & \\
\hline $\operatorname{BMI}\left(\mathrm{kg} / \mathrm{m}^{2}\right)$ & $27.625 \pm 4.4697$ & $28.102 \pm 4.1953$ & 0.3586 \\
\hline smoke more than 1 pack/day (yes) $\dagger$ & 0 & 0 & $>0.9999$ \\
\hline \multicolumn{4}{|l|}{ history nonop care (yes) } \\
\hline pain medication $\ddagger$ & $208(92.4)$ & $100(95.2)$ & 0.7169 \\
\hline \multicolumn{4}{|l|}{ opioid use§ } \\
\hline opium alkaloid & $27(12.0)$ & $7(6.7)$ & 0.1741 \\
\hline semisynthetic opioid derivative & $119(52.9)$ & $60(57.1)$ & 0.4794 \\
\hline synthetic opioid & $18(8.0)$ & $18(17.1)$ & 0.0215 \\
\hline physical therapy & $110(48.9)$ & $49(46.7)$ & 0.9290 \\
\hline collar & $27(12.0)$ & $15(14.3)$ & 0.6324 \\
\hline chiropractic & $61(27.1)$ & $23(21.9)$ & 0.5518 \\
\hline cervical traction & $45(20.0)$ & $21(20.0)$ & 0.6021 \\
\hline bed rest/immobilization & $110(48.9)$ & $49(46.7)$ & 0.6397 \\
\hline acupuncture & $18(8.0)$ & $6(5.7)$ & 0.4529 \\
\hline work status (being able to work) & $141(62.7)$ & $64(61.0)$ & $>0.9999$ \\
\hline driving status (being able to drive) & $210(93.3)$ & $102(97.1)$ & 0.4026 \\
\hline
\end{tabular}

cally significant differences were noted in neck pain at any other time points or arm pain at any time point. At 24 months, the mean change in neck pain from baseline was $54 \pm 25$ for the TDR group and $53 \pm 29$ for the ACDF group. Improvements in arm pain at 24 months were $35 \pm$ 35 for the TDR group and $34 \pm 38$ for the ACDF group.

\section{Patient Satisfaction}

Patient satisfaction was evaluated using a questionnaire, which asked whether patients were very satisfied, somewhat satisfied, somewhat dissatisfied, or very dissatisfied with the surgical treatment received. In both groups, the overall rates of patient satisfaction were high: at 24 months, 95.8\% of TDR patients and $92.0 \%$ of ACDF patients reported being either very or somewhat satisfied with their treatment. Patients were also asked if they would definitely, probably, probably not, or definitely not recommend the same surgical treatment to a friend with the same indications. At 24 months, $95.8 \%$ of patients in the TDR group and $88.5 \%$ of patients in the ACDF group reported that they would definitely or probably recommend the surgery to a friend.

Quality of Life (SF-12)

Patients in both groups were administered the SF-12 questionnaires and were evaluated separately on the phys-

TABLE 3: Perioperative data comparisons

\begin{tabular}{cccc}
\hline Measure & TDR $(n=234)^{*}$ & ACDF $(n=105)$ & p Value ${ }^{*}$ \\
\hline mean surgery time \pm SD $(\mathrm{hrs})$ & $2.2 \pm 0.8$ & $1.8 \pm 0.9$ & 0.0002 \\
mean blood loss \pm SD (ml) & $67.2 \pm 90.0$ & $70.3 \pm 78.78$ & 0.78 \\
mean hospitalization \pm SD (days) $\ddagger$ & $2.2 \pm 0.5$ & $2.4 \pm 2.1$ & 0.23 \\
\hline
\end{tabular}

\footnotetext{
* Number of patients in TDR group includes randomized (225) and nonrandomized training (9) patients.

† Unpaired t-test used to make comparisons across treatments.

$\ddagger$ Duration of hospitalization defined as: (date of discharge - date of surgery +1 day).
} 

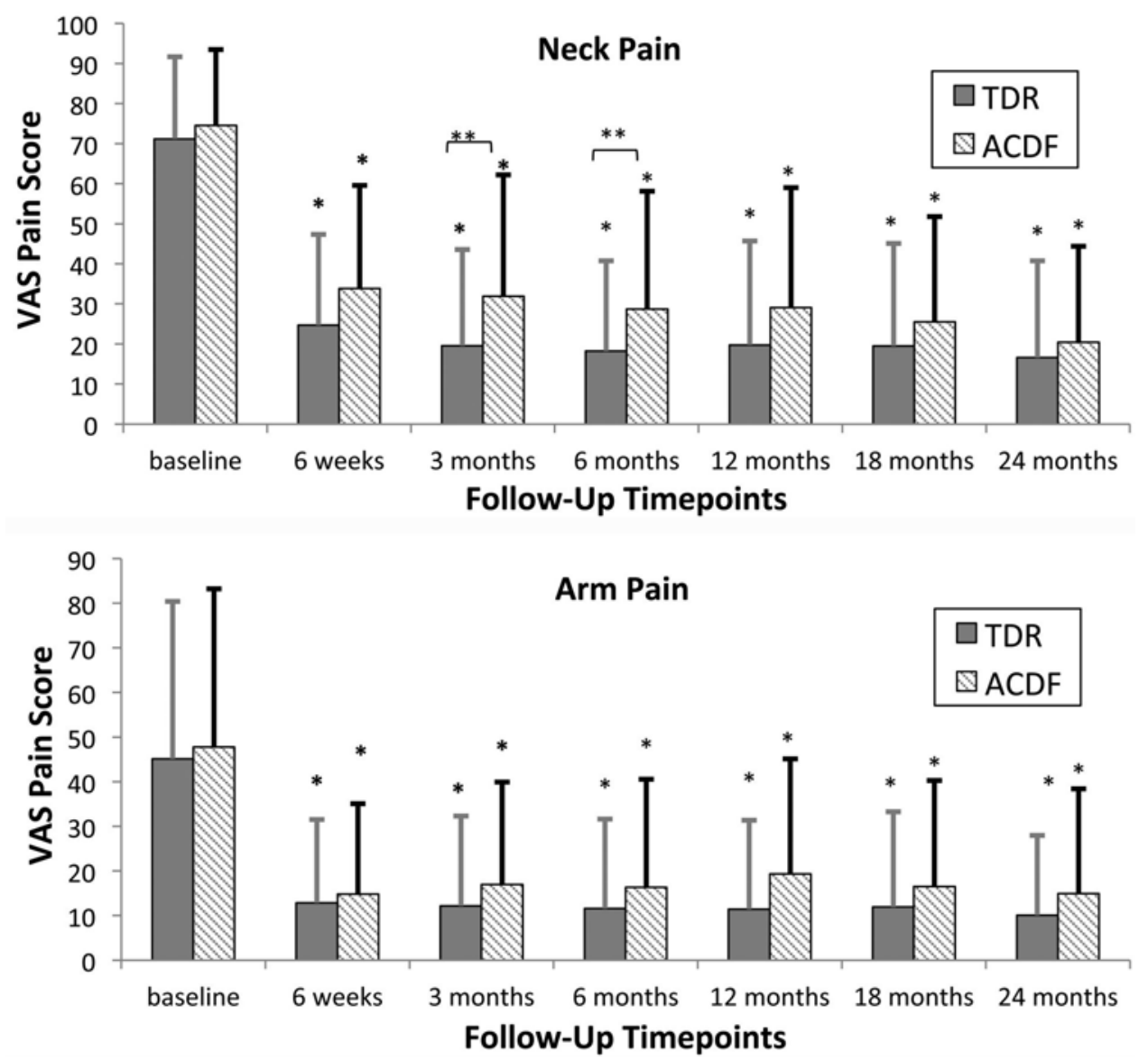

FIG. 3. Mean VAS neck (upper) and arm (lower) pain scores by time point. The VAS pain scores were collected at each follow-up visit. Error bars represent standard deviations. ${ }^{*} p<0.0001$, using the Wilcoxon signed-rank test to compare the change from baseline within treatment groups; ${ }^{* *} p<0.05$, using the unpaired t-test to compare the change from baseline value between treatments.

ical component summary (PCS) and mental component summary (MCS) scores. At baseline, the summary scores were statistically similar for the 2 study groups. As expected, patients in both groups improved postoperatively on both PCS and MCS scores. At 24 months, patients in the TDR group had mean PCS score improvement from baseline of 13.5 and mean improvement in MCS score of 9.5; patients in the ACDF group experienced a 10.5-point improvement in PCS score and 7.2-point improvement in MCS score. The difference between groups in terms of the change from baseline of the PCS score was statistically significant ( $p=0.03$ at 24 months) in favor of the TDR group at every time point (Fig. 4).

\section{Neck Disability Index}

The mean NDI scores were similar in both groups at baseline: $54 \pm 16$ for the TDR group and $55 \pm 15$ for the ACDF group. Patients in both groups showed marked improvement in NDI scores with significant improvements from baseline at all time points (Fig. 5). The mean improvement from baseline was significantly greater in the TDR group than the ACDF group at every time point (Fig. 5). Compared with baseline, the mean change in NDI at 24 months was $37 \pm 20$ in the TDR group and $30 \pm 19$ in the ACDF group. The NDI success rate in the TDR group was significantly higher at all time points $(\mathrm{p}<0.05)$, with 78.2\% of TDR patients and 61.8\% of ACDF patients considered a success on the NDI at 24 months. In both treatment groups, failure to meet the NDI success requirement was the greatest contributing factor to a patient's failure to meet the overall study success criteria.

\section{Subsequent Surgical Intervention}

Subsequent surgical interventions (classified as removal, revision, supplemental fixation, or reoperation) were defined as surgical procedures to modify the study device or surgeries at the index level for new or ongoing original indications. A total of 7 patients $(3.1 \%)$ in the TDR group and $12(11.4 \%)$ in the ACDF group required a subsequent surgical intervention through 24 months follow-up. The difference in the rates of subsequent surgical interventions between the 2 groups was statistically significant, with the TDR group requiring fewer subsequent surgical interventions. The absence of a subsequent surgical intervention was a requirement for overall study success and was a major factor contributing to the difference in overall study success rates.

In the ACDF group 12 patients required subsequent surgical intervention; there were 6 device removals, 1 revision, 3 supplemental fixations, and 2 reoperations. Nine 

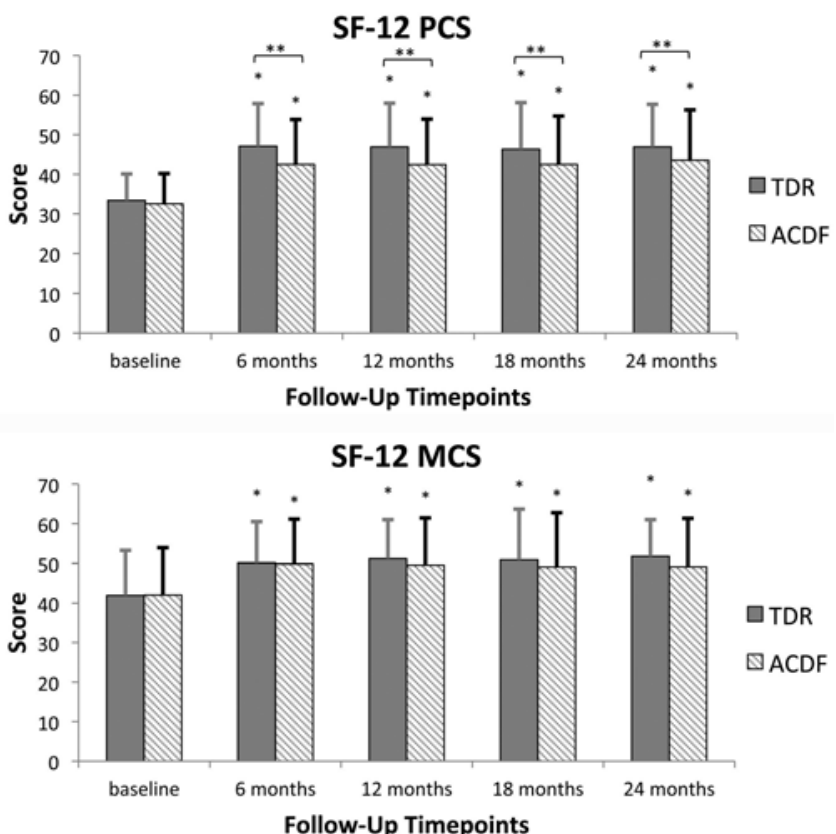

FIG. 4. Mean SF-12 PCS (upper) and MCS (lower) scores by time point. The SF-12 PCS and MCS scores were collected at baseline and at $6,12,18$, and 24 months postoperatively. Error bars represent standard deviations. ${ }^{*} p<0.0001$, using the Wilcoxon signed-rank test to compare the change from baseline. ${ }^{* *} p<0.05$, using the unpaired t-test to compare the change from baseline value between treatments.

of the surgical interventions were performed due to symptomatic pseudarthrosis or instability at 1 or both of the treated segments. In all subsequent surgeries in the ACDF group classified as removal, the classification was due to the removal of the control device, and in each case the fusion was either revised or extended to include an adjacent segment. The revision involved modification of the original hardware. The reoperations involved posterior surgery at 1 or both index levels, and supplemental fixation included posterior hardware at 1 or both index levels.

Seven patients in the TDR group required subsequent surgical intervention, including 4 removals, 2 reoperations, and 1 revision. One device was removed due to posterior migration of a single device component. At the time of the second surgery, the investigator confirmed the observations on radiography and CT review: the midline osteophyte resection on the superior endplate of the inferior adjacent vertebrae had been too aggressive. Much of the vertebral body posteriorly had been removed with subsequent migration and angulation of the TDR device. Due to this aggressive endplate resection, the surgeon elected to revise the TDR to fusion. Another removal in the TDR group was performed because the investigator reported that the "prosthesis failed to attach to endplates"; the investigator suggested that poor attachment was possibly due to overpreparation of the endplates resulting in insufficient bone contact. Another TDR was removed by a physician other than the study investigator for an unreported reason, and the final removal occurred because the patient continued to experience neck and arm pain. After all TDR removals, the treated segments were fused. Two reoperations were performed for patients in the TDR group: 1 was for facet spondylosis at both segments and 1 was for ongoing bilateral arm pain. The single revision, without removal, was performed to evacuate a hematoma 7 days after the index surgery, and during the procedure the surgeon repositioned the TDR device 1-2 mm posteriorly. No supplemental fixation procedures were performed for patients in the TDR group. There were also 2 patients in the TDR group who received surgical treatment at an adjacent level within 24 months of study surgery; however, these procedures did not result in study failure as they did not meet the definition of subsequent surgical interventions.

\section{Adverse Events}

Patients in the TDR group had a statistically significant lower incidence of device-related adverse events (identified by the CEC as definitely or possibly device related), with rates of $16.7 \%(39 / 225)$ and $34.3 \%$ (36/105) reported for TDR and ACDF patients, respectively. A summary of these device-related adverse events can be found in Table 4. The incidence of serious adverse events was also lower for the TDR group; through 24 months, the percentages of patients experiencing at least 1 serious adverse event were $23.9 \%$ and $32.4 \%$ for TDR and ACDF

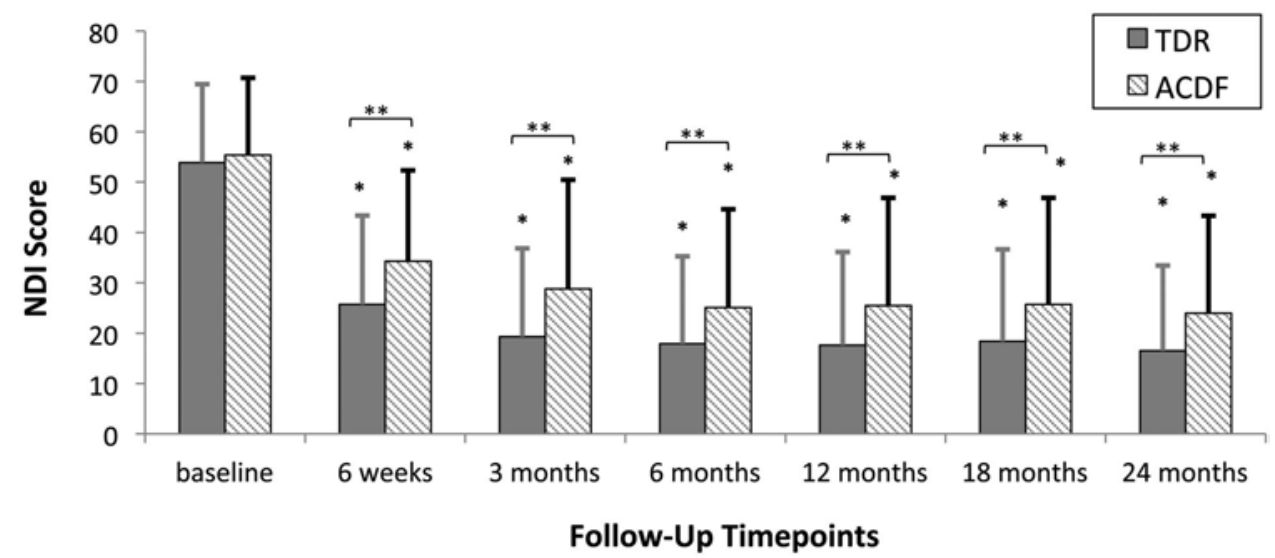

FIG. 5. Mean NDI score by time point. The NDI scores were collected at each visit. Error bars represent standard deviations. ${ }^{*} p<0.0001$, using the Wilcoxon signed-rank test to compare the change from baseline within treatment groups; ${ }^{* *} p<0.05$, using the unpaired t-test to compare the change from baseline between the 2 treatments. 
TABLE 4: Device-related adverse events according to the CEC*

\begin{tabular}{|c|c|c|c|c|}
\hline \multirow[b]{2}{*}{ Adverse Event } & \multicolumn{2}{|c|}{ TDR $(n=234) \dagger$} & \multicolumn{2}{|c|}{$\operatorname{ACDF}(n=105)$} \\
\hline & Events & Patients (\%) & Events & Patients (\%) \\
\hline anatomy/technical difficulty & 2 & $2(0.9)$ & 2 & $2(1.9)$ \\
\hline cervical (nonstudy surgery) & 1 & $1(0.4)$ & 1 & $1(1.0)$ \\
\hline cervical (study surgery) & 1 & $1(0.4)$ & 1 & $1(1.0)$ \\
\hline dysphagia/dysphonia & 10 & $9(3.8)$ & 9 & $8(7.6)$ \\
\hline dysphagia & 9 & $9(3.8)$ & 8 & $8(7.6)$ \\
\hline dysphonia & 1 & $1(0.4)$ & 1 & $1(1.0)$ \\
\hline gastrointestinal & 1 & $1(0.4)$ & 0 & 0 \\
\hline heterotopic ossification & 3 & $3(1.3)$ & 0 & 0 \\
\hline cervical (index level) & 2 & $2(0.9)$ & 0 & 0 \\
\hline cervical (adjacent level) & 1 & $1(0.4)$ & 0 & 0 \\
\hline malpositioned implant & 4 & $4(1.7)$ & 0 & 0 \\
\hline neck \&/or arm pain & 23 & $19(8.1)$ & 23 & $16(15.2)$ \\
\hline neck pain & 16 & $14(6.0)$ & 13 & $11(10.5)$ \\
\hline arm pain & 6 & $5(2.2)$ & 5 & $5(4.8)$ \\
\hline neck \& arm pain & 1 & $1(0.4)$ & 5 & $2(1.9)$ \\
\hline neurological & 12 & $10(4.3)$ & 24 & $11(10.5)$ \\
\hline upper extremity (sensory) & 7 & $6(2.6)$ & 12 & $4(3.8)$ \\
\hline neck & 2 & $2(0.9)$ & 7 & $6(5.7)$ \\
\hline upper extremity (reflex) & 1 & $1(0.4)$ & 1 & $1(1.0)$ \\
\hline upper extremity (motor) & 1 & $1(0.4)$ & 2 & $2(1.9)$ \\
\hline other & 0 & 0 & 1 & $1(1.0)$ \\
\hline back & 1 & $1(0.4)$ & 1 & $1(1.0)$ \\
\hline nonunion & 0 & 0 & 9 & $8(7.6)$ \\
\hline other & 0 & 0 & 2 & $2(1.9)$ \\
\hline other pain & 7 & $6(2.6)$ & 3 & $2(1.9)$ \\
\hline headache & 2 & $2(0.9)$ & 2 & $2(1.9)$ \\
\hline shoulder & 5 & $4(1.7)$ & 1 & $1(1.0)$ \\
\hline respiratory & 1 & $1(0.4)$ & 0 & 0 \\
\hline spinal event & 4 & $3(1.3)$ & 5 & $5(4.8)$ \\
\hline cervical (study surgery) & 4 & $3(1.3)$ & 5 & $5(4.8)$ \\
\hline trauma & 1 & $1(0.4)$ & 0 & 0 \\
\hline
\end{tabular}

groups, respectively. These serious adverse events did not necessarily involve the cervical spine and were frequently unrelated to the study device or surgery. Incidence rates for serious adverse events in the cervical spine definitely or possibly related to the device were $3.4 \%(n=8)$ in the TDR cohort and $14.3 \%(n=15)$ in the ACDF cohort. Absence of an adverse event classified as a major complication by the CEC was a requirement to meet overall study success criteria. Overall, $3.6 \%$ of patients in the TDR group and $6.7 \%$ of patients in the ACDF group had major complications associated with an adverse event.

\section{Neurological Status}

Neurological success, defined as the absence of significant neurological deterioration, was determined by investigator-conducted evaluations that included motor as- sessments of muscle strength, sensory assessments with pinprick and light touch, and reflex assessments that were traced to the nerve roots at the treated levels. At 24 months, $5.6 \%$ of the TDR group and $6.7 \%$ of the ACDF group had shown significant neurological deterioration, a statistically significant difference in favor of TDR as determined by the Farrington-Manning test to compare frequencies between treatments $(\mathrm{p}<0.0001)$.

\section{Radiographic Outcomes}

Device condition (in addition to motion analysis) was an important parameter measured as a radiographic outcome. Subsidence was considered to be significant if there was at least $3 \mathrm{~mm}$ of cranial or caudal motion of the device perpendicular to the vertebral endplates, and significant migration was defined as at least $3 \mathrm{~mm}$ of ante- 
rior or posterior motion of the device parallel to the vertebral endplates. Radiographic assessments revealed 1 case of posterior device migration in the TDR group, which is detailed above in the section on subsequent surgical intervention. Neither treatment group had any patients with significant device subsidence.

For the TDR group, radiographic success required nonfusion at both treated segments. Nonfusion was defined as $2^{\circ}$ or more of angular motion from flexion to extension or no radiographic evidence of bridging bone across the intervertebral space. At 24 months, $96.4 \%$ of the patients in the TDR group were classified as radiographically successful. In the ACDF group, radiographic success required evidence of fusion at both treated segments. The criteria for fusion included the following: less than $2^{\circ}$ of angular motion from flexion to extension, evidence of bridging bone across the intervertebral space, and radiolucent lines at no more than $50 \%$ of the graft-tovertebral interfaces. At 24 months, the overall radiographic success rate of the ACDF group was $79.8 \%$, with the remaining $20.2 \%$ (20 patients) classified as treatment failures. Every patient defined as a radiographic failure in the ACDF group had more than $2^{\circ} \mathrm{ROM}$ evident at 1 or both index levels on flexion/extension radiographs. The difference in radiographic success rates between the groups was statistically significant $(\mathrm{p}<0.0001)$ and favored the TDR study group.

Range of motion for each treated level was calculated quantitatively from lateral flexion/extension radiographs and anteroposterior right/left lateral bending radiographs. The ACDF group experienced the expected reduction in ROM in both flexion/extension and lateral bending. Mean $\mathrm{ROM}$ values at 24 months were less than $1^{\circ}$ for both the superior and inferior treated segments in both directions of movement. Mean flexion/extension ROM and lateral bending ROM for the TDR group was maintained throughout the study duration as compared with baseline. At the superior treated levels, mean ROM at 24 months was $10.1^{\circ} \pm 5.9^{\circ}$ in flexion/extension and $5.6^{\circ} \pm 3.3^{\circ}$ in lateral bending. For the inferior treated levels, the ROM values were $8.3^{\circ} \pm 5.3^{\circ}$ for flexion/extension and $5.4^{\circ} \pm$ $3.3^{\circ}$ in lateral bending (Fig. 6).

Adjacent-segment degeneration was evaluated using the Kellgren-Lawrence grading scale. If the degeneration grade for a segment increased by 1 or more points from the preoperative baseline, that segment was classified as having adjacent-level degeneration at the assessment time point. Using this criterion, at 12 months, $12.5 \%$ and $9.1 \%$ of ACDF patients had adjacent-level degeneration of the superior and inferior adjacent segments, respectively. At the same time point, $5.7 \%$ and $1.5 \%$ of TDR patients had degeneration at the superior and inferior adjacent segments, respectively. By 24 months, the differences between the ACDF and TDR groups were more dramatic. In the ACDF group, $33.3 \%$ and $18.1 \%$ of patients had degeneration at the superior and inferior segments, respectively. In the TDR group, adjacent-segment degeneration rates were $13.1 \%$ and $2.9 \%$ for the superior and inferior discs, respectively. The differences in adjacent-level degeneration rates were statistically significant in favor of the TDR group for both levels and at both the 12- and 24-month follow-ups $(p<0.03)$. Disc degeneration grades greater than 0 , which indicates at least minimal degeneration of the intervertebral disc, were noted more frequently at the superior adjacent segment than the inferior adjacent segment in both groups and at all time points, including the baseline assessment. Clinically relevant HO (Grades III \& IV) was noted in $11.5 \%$ of superior treated and $10.1 \%$ of inferior treated segments, with $3.7 \%$ and $2.8 \%$, respectively, classified as Grade IV HO at 24 months. Grade IV HO was present in 11 patients $(4.9 \%)$ at 24 months. Also at 24 months, $79.0 \%$ of superior and $70.6 \%$ of inferior segments were classified as stable, indicating no change in the $\mathrm{HO}$ grade since the 12-month assessments.

\section{Overall Clinical Success}

The composite measurement of overall study success included the following components: 1) functional improvement demonstrated by NDI success; 2) no subsequent surgical intervention at the index level; 3) no adverse event assessed by the CEC to be a major complication; 4) maintenance or improvement of neurological function; and 5) radiographic success. The overall success rates were $69.7 \%$ and $37.4 \%$ for the TDR and ACDF groups, respectively (Fig. 7, Table 5). The significantly higher overall success rate for the TDR group establishes both noninferiority and superiority as defined in the prospectively written statistical analysis plan. The TDR patients tended to achieve this success earlier and maintain it throughout the study. To demonstrate the effect of the radiographic requirement on overall study success rate, Fig. 7 also shows the success rates when only the 4 nonradiographic success criteria are considered. When only nonradiographic success criteria are considered, the success rate increases at every time point for both the TDR and ACDF groups compared to using all 5 criteria, with a larger increase in the ACDF group. However, even in this scenario, TDR maintains statistical superiority over ACDF at 24 months.

\section{Discussion}

Since its introduction more than 50 years ago, ACDF has become the standard surgical treatment for symptoms associated with cervical DDD. This procedure is also associated with the onset or progression of adjacent-segment degeneration. Over the past decade, a growing body of Level I evidence has demonstrated the potential of TDR as an alternative to ACDF, with studies focusing almost exclusively on single-level treatments. The study reported here was designed to evaluate the safety and effectiveness of 2-level TDR by testing for noninferiority of the Mobi$\mathrm{C}$ cervical artificial disc compared with 2-level ACDF. If noninferiority of Mobi-C was established, superiority was then tested. At the primary end point of 24 months, the overall study success rates were $69.7 \%$ and $37.4 \%$ for the TDR and ACDF groups, respectively, which met the noninferiority margin and established statistical superiority of the TDR treatment over ACDF.

In addition to statistical superiority in overall study success rate, 2-level TDR demonstrated significant advantages over 2-level ACDF in specific areas of clinical outcomes and complication rates. On average, the TDR patients experienced significantly greater improvement in NDI score and SF-12 PCS scores compared with baseline 

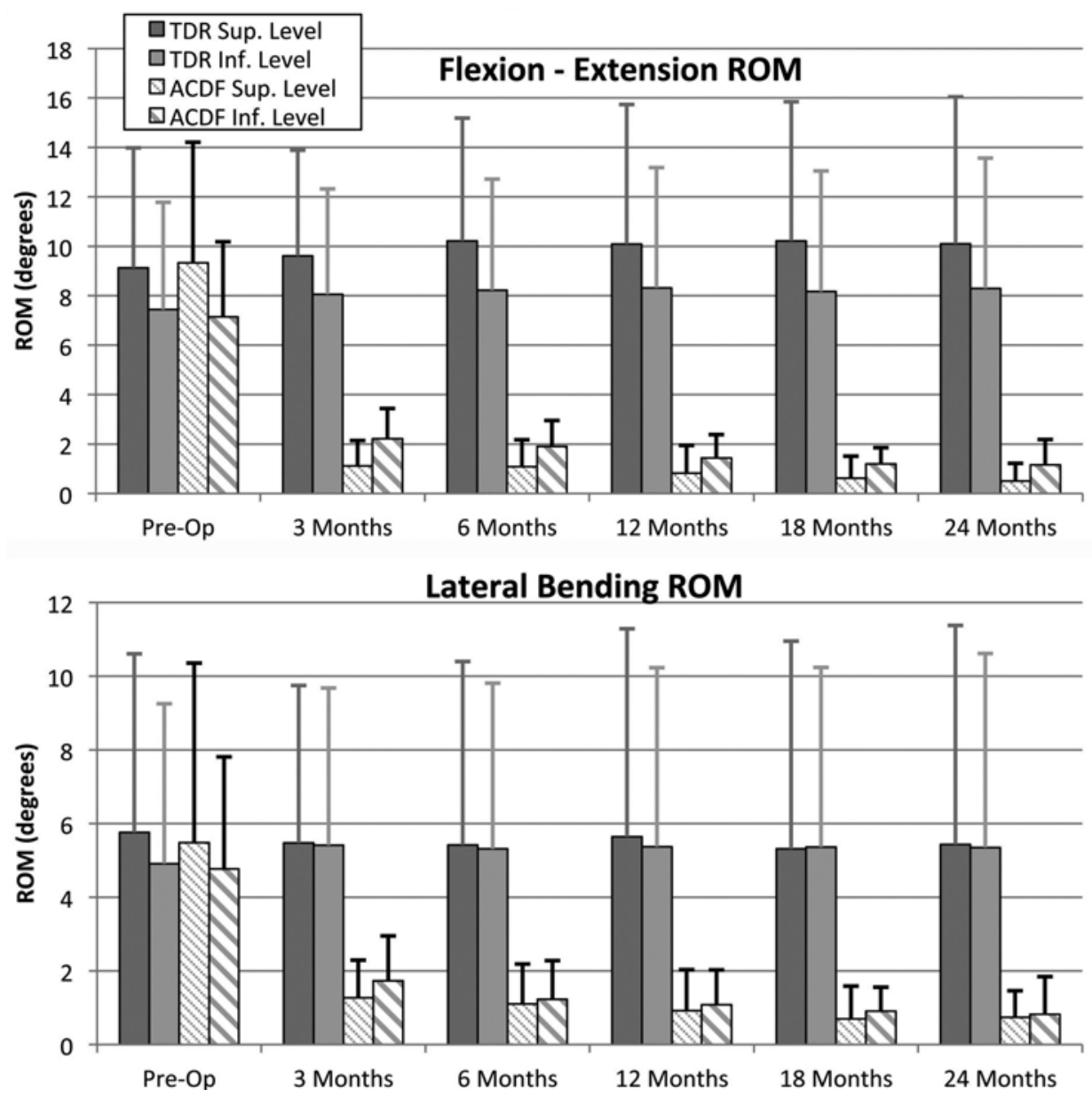

FIG. 6. Graphs of flexion-extension (upper) and lateral bending (lower) ROM by time point. Both graphs show mean ROM in degrees for both the superior (sup.) and inferior (inf.) treated levels. Error bars show standard deviation of the mean.

assessments at all time points in the study. The patients in the TDR group also showed a significantly greater improvement in neck pain at early time points. Patients in the TDR cohort required significantly fewer subsequent surgical interventions (3.1\%) compared with ACDF (11.4\%), had significantly lower rates of neurological deterioration, and had significantly lower rates of serious adverse events than patients in the ACDF cohort.

Grade IV HO was present in 11 TDR patients (4.9\%) at 24 months. Other TDR devices currently approved for 1-level use report 24-month Grade IV HO rates between $1.1 \%$ and $9.0 \% .^{16,17,37}$ The endplate design of the different TDR devices dictates the amount of vertebral endplate bone preparation, and it is theorized that more extensive vertebral endplate bone preparation is related to a higher incidence of HO. Mobi-C requires minimal vertebral endplate preparation due to its anatomical shape and small lateral teeth for fixation. Accordingly, the incidence of Grade IV HO was low (4.9\%), but the lack of NSAID use in the present study also likely affected $\mathrm{HO}$ rates. The HO rate could have possibly been lower in the Mobi-C patients if there had been no restrictions of NSAID use, similar to other TDR study protocols.

The success rate of the 2-level Mobi-C group (69.7\%) is comparable to the rate reported in the single-level arm of the Mobi-C Cervical Artificial Disc IDE study (73.6\%). ${ }^{38}$ This finding is consistent with those from studies that have compared single-level and multilevel TDR and found similar success rates. ${ }^{20,39,40}$ Pimenta et al. ${ }^{40}$ reported on a series of 229 consecutive patients treated at 1, 2, 3, or 4 levels with another TDR device. When compared with single-level patients, the multilevel patients showed no

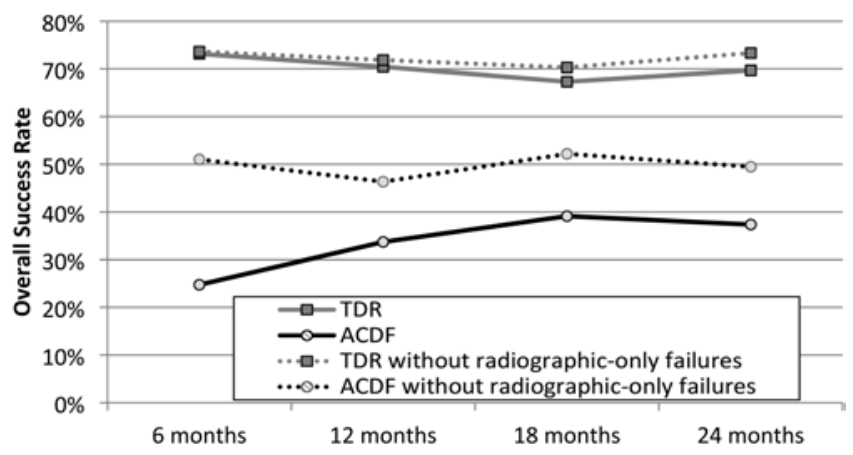

Fig. 7. Graph of overall study success rates by time point. Success rates are shown for both TDR and ACDF groups, with and without failures due only to radiographic major complications. Noninferiority is demonstrated at each time point for TDR compared with ACDF in both evaluations. 
Cervical total disc replacement with Mobi-C at 2 levels

TABLE 5: Overall study success per protocol and overall study success without radiographs

\begin{tabular}{cccc}
\hline Component & Randomized TDR & Randomized ACDF & $p$ Value* \\
\hline protocol overall success & $154 / 221(69.7 \%)$ & $37 / 99(37.4 \%)$ & $<0.0001$ \\
overall success w/out radiographs & $160 / 221(72.4 \%)$ & $49 / 99(49.5 \%)$ & $<0.0001$ \\
\hline * Using Farrington-Manning test to compare between the treatments. & &
\end{tabular}

increase in complications, similar improvements in pain and function, and similarly high study success rates (using Odom's criteria) at 3 years. This study suggests that TDR can be equally successful over multiple levels because each TDR segment is biomechanically independent from the adjacent segments. A biomechanical cadaver study by Phillips et al. ${ }^{39}$ supports this concept, demonstrating that 2-level TDR provides near-normal ROM at both operated segments without destabilizing the TDR or affecting motion at adjacent segments.

In contrast, the overall study success rate of the ACDF cohort in this study at 2 years was $37.4 \%$, which is much lower than the success rates reported for control ACDF cohorts in single-level TDR trials. The concurrently run single-level study arm of this IDE study reported a $65.3 \%$ overall study success rate for the ACDF cohort at 24 months $; 3$ the rates achieved in other single-level studies were similar. ${ }^{22,36,37}$ While this 2-level study arm used a definition of overall study success for the ACDF group that was identical to the single-level arm and similar to those used by other cervical arthroplasty trials, the low rate of overall study success achieved in the ACDF group does lead to a question: Are the criteria for overall study success more difficult to achieve for ACDF patients?

The definition of overall study success was prospectively planned and thus not subject to change, but 1 method of addressing this question is to closely examine the causes of study failure among ACDF patients. The first component to examine is the requirement for radiographic evidence of fusion for success for patients in the ACDF group. Table 5 includes overall success for each group categorized 2 ways: overall success per protocol (as described above) and overall success with the radiographs removed.

This analysis demonstrates that the criteria for radiographic evidence of a successful fusion are not the biggest factor contributing to failure in the ACDF group. Further analysis of failures reveals the most common causes of study failure among the ACDF patients are a failure to achieve NDI success and a patient requiring a subsequent surgical intervention.

The requirements of NDI success and the definition of subsequent surgical interventions were identical for all groups in this study, as well as for patients in the singlelevel arm of this study. Therefore, these requirements do not appear to be a particular disadvantage of the overall study success criteria that are artificially lowering the level of success in the multilevel ACDF patients compared with single-level patients and TDR patients. Despite the surprisingly low success rate of multilevel ACDF, this decrement in outcomes of multilevel ACDF compared with single-level ACDF is not without precedent. Other studies have also suggested that multilevel ACDF is less effective than single-level ACDF. In a retrospective review published by Swank et al., ${ }^{47}$ rates of pseudarthrosis were shown to increase from $10 \%$ for 1 -level ACDF to $44 \%$ for 2-level ACDF. Another study reported an increase in reoperation rates as number of treated levels increased. ${ }^{19} \mathrm{Re}-$ searchers have postulated a biomechanical basis for this decreasing efficacy as the number of fused segments is increased. ${ }^{10}$ As the number of fused levels increases, the length of the resulting rigid lever arm increases; this, in turn, amplifies the proportion of overall cervical motion that must be assumed by the adjacent segments, resulting in hypermobility and increased stress at the adjacent segments.

A widely held rationale for TDR success is a 2-part thesis: 1) the procedure maintains segmental mobility, and thus 2) TDR avoids the accelerated adjacent-segment degeneration. To the first point, the TDR cohort, on average, maintained preoperative mobility at both treated segments throughout the study period. Regarding adjacent-segment degeneration, when compared with the ACDF cohort, patients in the TDR cohort demonstrated significantly lower rates of radiographic adjacent-segment degeneration at both adjacent segments at 24 months. Continued observation will be needed to determine if this advantage in adjacent-level degradation translates to clinically relevant symptoms requiring surgical interventions.

This clinical trial demonstrated statistical superiority of 2-level TDR using the Mobi-C cervical artificial disc compared with 2-level ACDF at a primary end point of 2 years. Additional rigorous research will further the understanding of the safety and efficacy of multilevel cervical arthroplasty and arthrodesis procedures.

\section{Conclusions}

This FDA-regulated IDE clinical trial was designed to evaluate the safety and effectiveness of 2-level TDR using the Mobi-C cervical artificial disc compared with 2-level ACDF. Overall study success required that patients meet the criteria for 5 clinical and radiographic measures at the primary end point of 24 months. Using this composite scoring system, the overall study success rates were $69.7 \%$ and $37.4 \%$ for the TDR and ACDF groups, respectively, which established noninferiority and also demonstrated statistical superiority of the TDR group. Patients in the ACDF group were more than 3 times more likely to require a subsequent surgical intervention and twice as likely to have an adverse event related to the device when compared with the TDR patients. The TDR study group also had a significantly lower rate of serious adverse events. On average, the TDR study group maintained preoperative mobility at the treated segments, and the 2-year radiographic analysis indicated significantly less adjacent- 


\section{R. J. Davis et al.}

segment degeneration in this group. These data provide compelling Level I evidence in support of 2-level TDR as an alternative to 2-level ACDF in properly indicated patients.

\section{Disclosure}

Dr. Davis has served as a consultant for LDR, has received clinical or research support for this study from LDR, and has received support for statistical analysis and for study/writing or editorial assistance on this paper from LDR. Dr. Bae is a patent holder for LDR Spine, has invested in a private fund that holds equity in LDR Spine among other medical companies, has received research support from LDR Spine, and receives royalties for products from NuVasive, Stryker, and Zimmer Spine. Dr. Hisey serves as a consultant for LDR Spine, Zimmer Spine, DePuy/Synthes Spine, and Baxano Surgical, and is a patent holder for LDR Spine and Zimmer Spine. Dr. Kim is a patent holder for LDR, and is a consultant for LDR, Globus, and Stryker. Dr. Nunley has direct stock ownership in Amedica, Paradigm Spine, and Spineology; has received support of non-study-related clinical or research effort from the Cervical Spine Research Society, K2M, Biomet Spine, Axiomed, Medtronic, Nutech, NuVasive, Spinal Motion, and Vertiflex; is a patent holder for K2M and LDR Spine (specifically for the ROI-A Anterior lumbar interbody fusion cage); is a consultant for Nutech; and has received royalties, performed speaking and teaching arrangements, and served on the scientific advisory board of Osprey Biomedical and K2M. Dr. Peterson has direct stock ownership in LDR Spine. Dr. Stokes has direct stock ownership in LDR Spine and Diffusion Inc., and has received royalties from Genesys Orthopedics. Dr. Hoffman has direct stock ownership in, and serves as a consultant to, LDR and Nanovis. Dr. Rashbaum serves as a consultant to LDR and has received royalties and stock options from LDR. Dr. Gaede has received clinical or research support for this study from LDR.

Author contributions to the study and manuscript preparation include the following. Acquisition of data: all authors. Analysis and interpretation of data: Davis. Drafting the article: Davis, Kim. Critically revising the article: all authors. Reviewed submitted version of manuscript: all authors. Approved the final version of the manuscript on behalf of all authors: Davis.

\section{Acknowledgments}

LDR contributed to the design and conduct of the study and also provided assistance with analysis of data and manuscript review. The authors would like to thank the other principal investigators for their contributions to the study: Charlie Gordon, M.D., Texas Spine and Joint Hospital, Tyler, Texas; Arnold Schwartz, M.D., Orthopedic Spine Care of Long Island, Huntington Station, New York; Ali Araghi, M.D., The Core Institute, Phoenix, Arizona; David Tahernia, M.D., Desert Orthopedic Center, Rancho Mirage, California; Hazem Eltahawy, M.D., University Neurologic Systems, Wayne State University, Detroit, Michigan; Reginald Tall, M.D., Southeastern Clinical Research, Orlando, Florida; Douglas Wong, M.D., Panorama Orthopedics \& Spine Center, Golden, Colorado; Gerald Schell, M.D., St. Mary's of Saginaw, Field Neurosciences Institute, Saginaw, Michigan; Robert Jackson, M.D., Saddleback Memorial Medical Center, Laguna Hills, California; Michael Ramsey, M.D., West Texas Spine, Odessa, Texas; B. Christoph Meyer, M.D., Houston Orthopedic Hospital, Bellaire, Texas; Robert McLain, M.D., Cleveland Clinic, Cleveland, Ohio; Jon Park, M.D., Stanford Hospital and Clinics, Stanford, California; Ed Simmons, M.D., Simmons Orthopaedics and Spine Associates, Buffalo, New York; Mark Stern, M.D., California Institute of Neurological Surgery, Escondido, California; Phillip S. Yuan, M.D., Memorial Orthopaedic Surgical Group, Long Beach, California; and Guy O. Danielson III, M.D., Texas Spine and Joint Hospital, Tyler, Texas. The principal author would also like to thank Kelly Frank, M.S., of LDR Spine, Austin, Texas, for her assistance with data analysis and manuscript review.

\section{References}

1. Anderson PA, Rouleau JP: Intervertebral disc arthroplasty. Spine (Phila Pa 1976) 29:2779-2786, 2004

2. Brodke DS, Zdeblick TA: Modified Smith-Robinson procedure for anterior cervical discectomy and fusion. Spine (Phila Pa 1976) 17 (10 Suppl):S427-S430, 1992

3. Brown JA, Havel P, Ebraheim N, Greenblatt SH, Jackson WT: Cervical stabilization by plate and bone fusion. Spine (Phila Pa 1976) 13:236-240, 1988

4. Bui TL, Spector L, Odum S, Darden BV, Segabarth B, Patti J, et al: Adjacent segment degeneration following ProDisc-C Total Disc Replacement (TDR) and Anterior Cervical Discectomy and Fusion (ACDF): does surgeon bias effect radiographic interpretation? Presented at the annual meeting of the Cervical Spine Research Society, Chicago, 2012 (Abstract) (http:// www.csrs.org/web/searchAbstr/2012_Paper33.pdf) [Accessed July 8, 2013]

5. Carreon LY, Glassman SD, Campbell MJ, Anderson PA: Neck Disability Index, short form-36 physical component summary, and pain scales for neck and arm pain: the minimum clinically important difference and substantial clinical benefit after cervical spine fusion. Spine J 10:469-474, 2010

6. Chang UK, Kim DH, Lee MC, Willenberg R, Kim SH, Lim $\mathrm{J}$ : Range of motion change after cervical arthroplasty with ProDisc-C and prestige artificial discs compared with anterior cervical discectomy and fusion. J Neurosurg Spine 7:40-46, 2007

7. Coric D, Nunley PD, Guyer RD, Musante D, Carmody CN, Gordon CR, et al: Prospective, randomized, multicenter study of cervical arthroplasty: 269 patients from the Kineflex|C artificial disc investigational device exemption study with a minimum 2-year follow-up. Clinical article. J Neurosurg Spine 15:348-358, 2011

8. Croft P: An introduction to the Atlas of Standard Radiographs of Arthritis. Rheumatology (Oxford) 44 (Suppl 4):iv42, 2005

9. Cummins BH, Robertson JT, Gill SS: Surgical experience with an implanted artificial cervical joint. J Neurosurg 88:943948, 1998

10. Cunningham BW, Hu N, Zorn CM, McAfee PC: Biomechanical comparison of single- and two-level cervical arthroplasty versus arthrodesis: effect on adjacent-level spinal kinematics. Spine J 10:341-349, 2010

11. Davis RJ, Araghi A, Bae HW, Hisey MS, Nunley PD: Comparison of complication rates associated with two level cervical arthroplasty versus two level anterior cervical discectomy and fusion. Spine J 12 Suppl:S139-S140, 2012 (Abstract)

12. Davis RJ, Araghi A, Bae HW, Hisey MS, Nunley PD: Investigational device exemption trial of cervical arthroplasty for treatment of degenerative disc disease at two levels: 24-month results of 330 subjects. Spine J 12 Suppl:S95-S96, 2012 (Abstract)

13. Davis RJ, Bae HW, Gaede SE, Hisey MS, Kim KD, Nunley PD, et al: Radiographic outcomes including adjacent level degeneration of FDA investigational device trial comparing multi-level use of Mobi-C Cervical Artificial Disc to anterior discectomy and fusion. Presented at the annual meeting of the Cervical Spine Research Society, Scottsdale, AZ, 2011 (Abstract) (http://www.csrs.org/web/searchAbstr/2011_Paper68. pdf) [Accessed July 8, 2013]

14. Dmitriev AE, Cunningham BW, Hu N, Sell G, Vigna F, McAfee PC: Adjacent level intradiscal pressure and segmental kinematics following a cervical total disc arthroplasty: an in vitro human cadaveric model. Spine (Phila Pa 1976) 30:11651172,2005

15. Fijn R, Koorevaar RT, Brouwers JR: Prevention of heterotopic ossification after total hip replacement with NSAIDs. Pharm World Sci 25:138-145, 2003

16. Food and Drug Administration: Summary of Safety and Effectiveness Data: PCM Cervical Disc. (http://www.access 
data.fda.gov/cdrh_docs/pdf10/P100012b.pdf) [Accessed June 19, 2013]

17. Food and Drug Administration: Summary of Safety and Effectiveness Data: SECURE-C Cervical Disc. (http://www. accessdata.fda.gov/cdrh_docs/pdf10/P100003b.pdf) [Accessed June 19, 2013]

18. Fraser JF, Härtl R: Anterior approaches to fusion of the cervical spine: a metaanalysis of fusion rates. J Neurosurg Spine 6:298-303, 2007

19. Geisler FH, Caspar W, Pitzen T, Johnson TA: Reoperation in patients after anterior cervical plate stabilization in degenerative disease. Spine (Phila Pa 1976) 23:911-920, 1998

20. Goffin J, Van Calenbergh F, van Loon J, Casey A, Kehr P, Liebig $\mathrm{K}$, et al: Intermediate follow-up after treatment of degenerative disc disease with the Bryan Cervical Disc Prosthesis: singlelevel and bi-level. Spine (Phila Pa 1976) 28:2673-2678, 2003

21. Harrod CC, Hilibrand AS, Fischer DJ, Skelly AC: Adjacent segement pathology following cervical motion-sparing procedures or devices compared with fusion surgery. Spine (Phila Pa 1976) 37 (22 Suppl):S96-S112, 2012

22. Heller JG, Sasso RC, Papadopoulos SM, Anderson PA, Fessler RG, Hacker RJ, et al: Comparison of BRYAN cervical disc arthroplasty with anterior cervical decompression and fusion: clinical and radiographic results of a randomized, controlled, clinical trial. Spine (Phila Pa 1976) 34:101-107, 2009

23. Hilibrand AS, Robbins M: Adjacent segment degeneration and adjacent segment disease: the consequences of spinal fusion? Spine J 4 (6 Suppl):190S-194S, 2004

24. Hsu WK: The rationale for cervical disc arthroplasty. Tech Orthop 25:84-87, 2010

25. Jaramillo-de la Torre JJ, Grauer JN, Yue JJ: Update on cervical disc arthroplasty: where are we and where are we going? Curr Rev Musculoskelet Med 1:124-130, 2008

26. Jawahar A, Cavanaugh DA, Kerr EJ III, Birdsong EM, Nunley PD: Total disc arthroplasty does not affect the incidence of adjacent segment degeneration in cervical spine: results of 93 patients in three prospective randomized clinical trials. Spine J 10:1043-1048, 2010

27. Kellgren JH, Lawrence JS: Radiological assessment of osteoarthrosis. Ann Rheum Dis 16:494-502, 1957

28. Kellgren JH, Lawrence JS, Bier F: Genetic factors in generalized osteo-arthrosis. Ann Rheum Dis 22:237-255, 1963

29. Kjaersgaard-Andersen P, Schmidt SA: Total hip arthroplasty. The role of antiinflammatory medications in the prevention of heterotopic ossification. Clin Orthop Relat Res (263):78-86, 1991

30. Kulkarni V, Rajshekhar V, Raghuram L: Accelerated spondylotic changes adjacent to the fused segment following central cervical corpectomy: magnetic resonance imaging study evidence. J Neurosurg 100 (1 Suppl Spine):2-6, 2004

31. Lawrence BD, Hilibrand AS, Brodt ED, Dettori JR, Brodke DS: Predicting the risk of adjacent segment pathology in the cervical spine: a systematic review. Spine (Phila Pa 1976) 37 (22 Suppl):S52-S64, 2012

32. Lopez-Espina CGAF, Amirouche F, Havalad V: Multilevel cervical fusion and its effect on disc degeneration and osteophyte formation. Spine (Phila Pa 1976) 31:972-978, 2006

33. McAfee PC, Cunningham BW, Devine J, Williams E, Yu-Yahiro J: Classification of heterotopic ossification $(\mathrm{HO})$ in artificial disk replacement. J Spinal Disord Tech 16:384-389, 2003

34. McMahon JS, Waddell JP, Morton J: Effect of short-course indomethacin on heterotopic bone formation after uncemented total hip arthroplasty. J Arthroplasty 6:259-264, 1991

35. Mehren C, Suchomel P, Grochulla F, Barsa P, Sourkova P, Hradil J, et al: Heterotopic ossification in total cervical artificial disc replacement. Spine (Phila Pa 1976) 31:2802-2806, 2006

36. Mummaneni PV, Burkus JK, Haid RW, Traynelis VC, Zdeblick T: Clinical and radiographic analysis of cervical disc arthroplasty compared with allograft fusion: a randomized controlled clinical trial. J Neurosurg Spine 6:198-209, 2007
37. Murrey D, Janssen M, Delamarter R, Goldstein J, Zigler J, Tay $\mathrm{B}$, et al: Results of the prospective, randomized, controlled multicenter Food and Drug Administration investigational device exemption study of the ProDisc-C total disc replacement versus anterior discectomy and fusion for the treatment of 1-level symptomatic cervical disc disease. Spine J 9:275-286, 2009

38. Ohnmeiss D, Hisey MS, Bae H, Davis RJ, Hoffman GA, Kim $\mathrm{K}$, et al: Multicenter, prospective, randomized, controlled investigational device exemption study comparing Mobi $\mathrm{C}$ Cervical Artificial Disc to anterior fusion in the treatment of symptomatic cervical degenerative disc disease. Spine J 11 Suppl:S16-S17, 2011 (Abstract)

39. Phillips FM, Tzermiadianos MN, Voronov LI, Havey RM, Carandang G, Dooris A, et al: Effect of two-level total disc replacement on cervical spine kinematics. Spine (Phila Pa 1976) 34:E794-E799, 2009

40. Pimenta L, McAfee PC, Cappuccino A, Cunningham BW, Diaz R, Coutinho E: Superiority of multilevel cervical arthroplasty outcomes versus single-level outcomes: 229 consecutive PCM prostheses. Spine (Phila Pa 1976) 32:1337-1344, 2007

41. Pracyk JB, Traynelis VC: Treatment of the painful motion segment: cervical arthroplasty. Spine (Phila Pa 1976) 30 (16 Suppl):S23-S32, 2005

42. Rhee JM: Cervical arthroplasty: a success, failure, or both? Spine J 10:731-732, 2010

43. Rietman CA, Hipp JA, Nguyen L, Esses S: Changes in segmental intervertebral motion adjacent to cervical arthrodesis: a prospective study. Spine (Phila pa 1976) 29:E221-E226, 2004

44. Robertson JT, Papadopoulos SM, Traynelis VC: Assessment of adjacent-segment disease in patients treated with cervical fusion or arthroplasty: a prospective 2-year study. J Neurosurg Spine 3:417-423, 2005

45. Sell S, Willms R, Jany R, Esenwein S, Gaissmaier C, Martini $\mathrm{F}$, et al: The suppression of heterotopic ossifications: radiation versus NSAID therapy-a prospective study. J Arthroplasty 13:854-859, 1998

46. Smith GW, Robinson RA: The treatment of certain cervicalspine disorders by anterior removal of the intervertebral disc and interbody fusion. J Bone Joint Surg Am 40-A:607-624, 1958

47. Swank ML, Lowery GL, Bhat AL, McDonough RF: Anterior cervical allograft arthrodesis and instrumentation: multilevel interbody grafting or strut graft reconstruction. Eur Spine J 6:138-143, 1997

48. Upadhyaya CD, Wu JC, Trost G, Haid RW, Traynelis VC, Tay B, et al: Analysis of the three United States Food and Drug Administration investigational device exemption cervical arthroplasty trials. Clinical article. J Neurosurg Spine 16:216-228, 2012

49. Zindrick M, Harris MB, Humphreys SC, O'Leary PT, Schneiderman G, Watters WC III, et al: Cervical disc arthroplasty. J Am Acad Orthop Surg 18:631-637, 2010

Manuscript submitted June 1, 2012.

Accepted June 17, 2013.

Results from this study were previously presented at the North American Spine Society Meeting in 2011 and 2012, International Society for Advancement of Spine Surgery Meeting in 2010, Cervical Spine Research Society Meeting in 2011 and 2012, Eurospine in 2011, and AANS/CNS Joint Spine Section Meeting in 2011.

Please include this information when citing this paper: published online September 6, 2013; DOI: 10.3171/2013.6.SPINE12527.

Address correspondence to: Reginald J. Davis, M.D., Greater Baltimore Neurosurgical Associates, Department of Neurosurgery, 6535 N. Charles St., Ste.600, Baltimore, MD 21204. email: rjdavismd@ aol.com. 